\title{
Childhood Extraocular Retinoblastoma
}

National Cancer Institute

\section{Source}

National Cancer Institute. Childhood Extraocular Retinoblastoma. NCI Thesaurus. Code C9048.

Retinoblastoma during childhood that has spread beyond the eye. 